\title{
COVER-PRESERVING EMBEDDING OF MODULAR LATTICES
}

\author{
E. FRIED AND E. T. SCHMIDT
}

\begin{abstract}
In this note we prove: If a subdirect product of finitely many finite projective geometries has the cover-preserving embedding property, then so does each factor.
\end{abstract}

In what follows all the lattices will be finite modular ones. A finite lattice $K$ has the cover-preseving embedding property, abbreviated as CPEP with respect a variety $V$ of lattices if whenever $K$ can be embedded into a finite lattice $L$ in $V$, then $K$ has a cover-preserving embedding into $L$, that is an embedding $f$ with the property that if $a$ covers $b$ in $K$ then $f(a)$ covers $f(b)$ in $L$. In a paper of E. Fried, G. Grätzer and H. Lakser, [1] it was proved that a finite projective geometry has the cover-preserving embedding property with respect to the variety $M$ of all modular lattices if and only if one of the following three conditions hold: (i) the length of $P$ is 1 ; (ii) the length of $P$ is 2 and $P$ is isomorphic to $\mathbf{M}_{3}$; (iii) the length of $P$ is greater then 2 and either $P$ is non-arguesian or $P$ is arguesian and for some prime $p$ each interval of $P$ of length 2 contains $p+1$ atoms (i.e. $P$ is a projective geometry over a prime field). Later in E. T. Schmidt, [2] the following theorem was formulated:

Theorem 1. If a finite modular lattice $L$ has the CPEP with respect to $M$ then $L$ is the subdirect product of projective geometries of type (i)-(iii).

Really in [2] the following was proved: If a finite modular lattice $L$ has the CPEP with respect to $M$ then $L$ is the subdirect product of projective geometries. The proof that the subdirect components are just the projective geometries (i)-(iii) was missing. This statment seems in the first moment quite trivial, but it is far not so.

Date: April 2, 2000.

1991 Mathematics Subject Classification. Primary 06B10; Secondary 06D05.

Key words and phrases. Cover-preserving sublattice, modular lattice, subdirect product, projective geometry.

The research of both authors was supported by the Hungarian National Foundation for Scientific Research, under Grant No. 1903. 
In this short note we prove that the factors in Theorem 1 are projective geometries of the given type, indeed. First of all we formulate an interesting property of the finite complemented modular lattices. It is easy to see: if $\phi: M \mapsto P$ is a surjection of a finite modular lattice $M$ onto a complemented modular lattice $P$, then $M$ contains an interval $P^{\prime}$ such that the restriction of $\phi$ to $P^{\prime}$ is an isomorphism between $P^{\prime}$ and $P$. If $P$ is a projective geometry then we can prove somewhat more:

Lemma 1. Let $M$ be any finite modular lattice, $P$ a finite projective geometry with the bounds 0 and 1 and $\phi: M \mapsto P$ a surjective homomorphism. Then, we have:

1. There exists a (unique) $a \in M$ with $\phi(a)=0$ such that $\phi(x)=0$ implies $x \leq a$.

2. There exists a (unique) $b \in M$ with $\phi(b)=1$ and $b \geq a$ such that $\phi(y)=1$ and $y \geq a$ together imply $y \geq b$.

3. $\phi \uparrow[a, b]$ is an isomorphism.

The interval $[a, b]$ will be called the "natural coimage" of $\phi$.

Proof. 1. The set $\{x ; \phi(x)=0\}$ is an ideal which is principal by finitness. If it is generated by $a$, then $a$ has the desired property.

2. The set $\{y ; \phi(y)=1, y \geq a\}$ is a filter which is principal by finitness. If it is generated by $b$, then $b$ has the desired property.

3. The restriction $\psi=\phi \uparrow[a, b]$ is obviously onto. Suppose $x=$ $\psi(u)=\psi(v)$. We may suppose $u \leq v$, as well. Let $y$ be a complement of $x$. By surjectivity, we have a $w \in[a, b]$ such that $\psi(w)=y$. Then

$$
\psi(v \wedge w)=0 \quad \text { and } \quad \psi(u \vee w)=1
$$

(1) and (2) imply $v \wedge w=a$ and $u \vee w=b$, respectively. Hence, by modularity, $u=v$.

Corollary 1. Let $\phi: M \mapsto P$ as in Lemma 1 and let $\chi: M \mapsto K$ be another homomorphism to the lattice $K$. Then the restriction of $\chi$ maps the natural coimage $[a, b]$ of $\phi$ either to a single element of $K$ or this restriction is one-to-one on $[a, b]$.

Lemma 2. Let $L$ be a subdirect product of the finite projective geometries $P_{i}$ together with the natural projections $\phi_{i}: L \mapsto P_{i}$ and with the natural coimages $\left[a_{i}, b_{i}\right],(i \in\{1,2, \ldots, n\})$. Suppose, this is a shortest decomposition. Then, $\phi_{i}$ maps $\left[a_{j}, b_{j}\right]$ to a single element, for $i \neq j$.

Proof. Suppose, say, that $\phi_{2}$ does not map $\left[a_{1}, b_{1}\right]$ to a single element. Then by Corollary $1, \phi_{2} \uparrow\left[a_{1}, b_{1}\right]$ is one-to-one. 
We are going to show, that in this case we may omit $P_{1}$ from the subdirect product. In other words, for $x \in L$ the mapping

$$
\psi_{1}: x \mapsto\left(\phi_{2}(x), \ldots, \phi_{n}(x)\right)
$$

is one-to-one.

Let $x \neq y$ be elements of $L$. If $\phi_{1}(x)=\phi_{1}(y)$, then for some $i$ we have $\phi_{i}(x) \neq \phi_{i}(y)$, i.e., $\psi_{1}(x) \neq \psi_{1}(y)$. Otherwise,

$$
\phi_{1}\left(\left(a_{1} \vee x\right) \wedge b_{1}\right)=\phi_{1}(x) \neq \phi_{1}(y)=\phi_{1}\left(\left(a_{1} \vee y\right) \wedge b_{1}\right),
$$

hence, by our condition, $\psi_{1}\left(\left(a_{1} \vee x\right) \wedge b_{1}\right) \neq \psi_{1}\left(\left(a_{1} \vee y\right) \wedge b_{1}\right)$. Therefore, we must have $\psi_{1}(x) \neq \psi_{1}(y)$, as well.

Corollary 2. Let $L$ be a subdirect product of the projective geometries $P_{i}$ together with the natural projections $\phi_{i}: L \mapsto P_{i}$ and with the natural coimages, $\left[a_{i}, b_{i}\right],(i \in\{1,2, \ldots, n\})$. Suppose, this is a shortest decomposition. Let, further, $\psi: L \mapsto K$ a homomorphism which sends $\left[a_{i}, b_{i}\right]$ onto $K$ for some $i$. Then $\psi$ sends all $\left[a_{j}, b_{j}\right]$ to a single element for each $j \neq i$.

Proof. We have by, Lemma 2,

$$
\phi_{i}\left(\left(a_{i} \vee a_{j}\right) \wedge b_{i}\right)=\phi_{i}\left(a_{j}\right)=\phi_{i}\left(b_{j}\right)=\phi_{i}\left(\left(a_{i} \vee b_{j}\right) \wedge b_{i}\right),
$$

hence, $\left(a_{i} \vee a_{j}\right) \wedge b_{i}=\left(a_{i} \vee b_{j}\right) \wedge b_{i}$, since $\phi_{i}$ is one-to-one on $\left[a_{i}, b_{i}\right]$, yielding

$$
\begin{aligned}
& \psi\left(a_{j}\right)=\left(\psi\left(a_{i}\right) \vee \psi\left(a_{j}\right)\right) \wedge \psi\left(b_{i}\right)=\psi\left(\left(a_{i} \vee a_{j}\right) \wedge b_{i}\right) \\
= & \left.\psi\left(\left(a_{i} \vee b_{j}\right)\right) \wedge b_{i}\right)=\left(\psi\left(a_{i}\right) \vee \psi\left(b_{j}\right)\right) \wedge \psi\left(b_{i}\right)=\psi\left(b_{j}\right) .
\end{aligned}
$$

This finishes the proof of the Corollary.

Lemma 3. Let $L$ be an irreducible subdirect product of the finite projective geometries $P_{1}, \ldots, P_{t}$. If one of the factors fails the CPEP, then so does $L$.

Proof. We arrange the factors so that the first $s$ fails CPEP and the other $t-s$ satisfies it. Let $A_{i}$ denote the number of atoms in $P_{i}$. We choose $P_{1}$ so that it has the highest dimension among the first $s$ component and, that $A_{1} \geq A_{2}$ for $i \leq s$ if $\operatorname{dim}\left(P_{i}\right)=\operatorname{dim}\left(P_{1}\right)$. We arrange the first $s$ factors so that $P_{1}, \ldots, P_{r}$ are isomorphic to $P_{1}$ and $P_{r+1}, \ldots, P_{s}$ are non-isomorphic to $P_{1}$. By our assumption there exist a lattice $Q_{1}$ such that $P_{1}$ has an embedding into it but $P_{1}$ has no cover-preserving embedding into it.

Now, we define $Q_{i}=Q_{1}$ for $i \leq r$ and $Q_{i}=P_{i}$ for $i>r$, and consider the direct product

$$
\widehat{L}=Q_{1} \times Q_{2} \times \ldots \times Q_{r} \times Q_{r+1} \times \ldots \times Q_{t} .
$$


$L$ has an obvious embedding into $\widehat{L}$. We prove that $L$ has no coverpreserving embedding into $\widehat{L}$. Assume, by way of contradiction, that $g: L \mapsto \widehat{L}$ is a cover-preserving embedding. Then, the restriction of $g$ to each natural coimage $\left[a_{i}, b_{j}\right]$ is a cover-preserving embedding, as well. Let $g_{j}$ denote the embedding $g$ followed by the $j$-th projection of $\widehat{L}$. By Corollary 1., the restriction of $g_{j}$ is either a cover-preserving embedding of $\left[a_{i}, b_{i}\right]$ into $Q_{j}$ or it sends this interval to a single element. Since $g$ is an embedding and $\widehat{L}$ is written as a direct product, forevery $i$ must exit a $j$ such that $g_{j}$ yields a cover-preserving embedding of $\left[a_{i}, b_{i}\right]$ into $Q_{j}$. However, we are going to prove that this is is impossible for $i=1$. We have to distinguish some cases.

Case 1. $j \leq r$. By our choice, $\left[a_{1}, b_{1}\right] \cong P_{1}$ has no cover-preserving embedding into $Q_{1}$.

Case 2. $r<j \leq s$ and $\operatorname{dim}\left(P_{j}\right)<\operatorname{dim}\left(P_{1}\right)$. Then, $Q_{j}=P_{j}$, hence $P_{1}$ cannot be a sublattice of $P_{j}$.

Case 3. $r<j \leq s$. Let $\operatorname{dim}\left(P_{k}\right) \geq \operatorname{dim}\left(P_{1}\right)$. Then, by our choice, we must have $\operatorname{dim}\left(P_{j}\right)=\operatorname{dim}\left(P_{1}\right)$. However, in this case we have $A_{j} \leq A_{1}$. If $A_{j}<A_{i}$, then $P_{1}$ has no embedding into $P_{j}$, whereas $A_{j}=A_{1}$ yealds $P_{j} \cong P_{1}$, i.e., $j \leq r$, which was discussed in Case 1 .

Case $4 . j>s$. Since $Q_{j}=P_{j}$ inthis case, there exist isomorhisms $h_{j}: Q_{j} \longrightarrow\left[a_{j}, b_{j}\right]$ for $(j=s+1, \ldots, t)$. Let, further, $k_{j}$ denote the restriction $g_{j} \uparrow\left[a_{j}, b_{j}\right]$. By $j . s$ (i.e., by $Q_{j}=P_{j}$ ) and by Corollary 1. , if $\operatorname{Im}\left(k_{j}\right)$ has more than one element, then $g_{j}$ maps all the other $\left[a_{i}, b_{i}\right]$ to a single element. In other words:

$(\star)$ : If $g_{j}$ maps $\left[a_{i}, b_{i}\right]$ isomorphically into $Q_{j}$, then $k_{j}$ is trivial (i.e., maps to a single element).

(In what follows, we shall use the notation $g_{j}$ for the restriction $g_{j} \uparrow\left[a_{n}, b_{n}\right]$, as well, provided that the image of this interval has more then one element.)

Now, we have the cover-preserving embedding $\left[a_{1}, b_{1}\right] \longrightarrow P_{j_{1}}$. Then, by $(\star) k_{j_{1}}$ is trivial. Hence, we must have a $g_{j_{2}}$ embedding $\left[a_{j_{1}}, b_{j_{2}}\right]$ into $Q_{j_{2}}$. If $j_{2}>s$, then we can continue our procedure. Corollary 1 . assure that this chain cannot close, that is there exists an $n$, such that $j_{n} \leq s$. Now, consider the diagram:

$\left[a_{1}, b_{1}\right] \stackrel{g_{j_{1}}}{\longrightarrow} Q_{j_{1}} \stackrel{h_{j_{1}}}{\longrightarrow}\left[a_{j_{1}}, b_{j_{1}}\right] \stackrel{g_{j_{2}}}{\longrightarrow} Q_{j_{2}} \stackrel{h_{j_{2}}}{\longrightarrow} \ldots \stackrel{h_{j_{n-1}}}{\longrightarrow}\left[a_{j_{n-1}}, b_{j_{n-1}}\right] \stackrel{g_{j_{n}}}{\longrightarrow} Q_{j_{n}}$.

Here, the first, third, etc. mappings are cover-preserving embeddings whereas the second, fourth, etc are isomorphisms. Hence, their product yields a cover-preserving embedding of $\left[a_{1}, b_{1}\right]$ into $Q_{j_{n}}$ for some $j_{n} \leq s$ contradicting one of the first three cases. 
Remarks.1.Some results of this paper remain valid for modular lattices of finite length.

2.The Theorem gives only a necessary condition for modular lattices to have (CPEP). It seems to be very complicated to characterize the finite distributive lattices satisfying (CPEP).

\section{REFERENCES}

[1] E. Fried,G. Grätzer and H. Lakser, Projective geometries as cover-preserving sublattices, Algebra Universalis.

[2] E.T. Schmidt, Cover-preserving embedding, Periodica Mathematica Hungarica, 23 (1991), 17-24.

EÖTVÖs UNIVERSITY OF BUDAPEST

Department of Algebra and Numbertheorie

Budapest, Muzeum-KRT. 6-8

HUNGARY

E-mail address: efried@ludens.elte.hu

TeChnical University of Budapest

TRANSPORT ENGINEERING FACULTY

Department of Mathematics

1111 BUDAPEST

MÜEGYETEM RKP. 9

HUNGA RY

E-mail address: h1175sch@ella.hu 